\title{
Reflexiones sobre la transparencia estructural
}

\author{
Oscar Linares de la Torre, Albert Albareda Valls \\ Recibido 2019.02.20 :.: Aceptado 2019.03.11 \\ DOI: 10.5821/palimpsesto.19.7047
}

ABSTRACT

Desde la antigüedad hasta el siglo XIX la estructura portante tiene un papel fundamental en la definición del espacio arquitectónico. Sin embargo, la irrupción del acero como material estructural y del vidrio como cerramiento transparente durante el siglo XIX permite disociar ambas funciones en dos
sistemas constructivos totalmente diferentes. La arquitectura moderna, a través de la cubierta plana y la planta libre, hace de esta dicotomía su estandarte: nunca antes en la historia de la arquitectura, la estructura vertical había adquirido una autonomia total con respecto al cerramiento, pudiendo éste ser totalmente transparente. La voluntad de disponer de cerramientos de vidrio de cada vez mayores dimensiones, hace que la industria del vidrio evolucione considerablemente a finales del siglo XX. Esta evolución tecnológica deriva en una mejora importantísima de a plantear su uso como elemento estructural: por primera vez en la historia de la construcción es posible emplear un material transparente como estructura. Estructura y cerramiento vuelven a unificarse como en la antigüedad, pero esta vez lo hacen en un elemento totalmente transparente. La estructura, entendida como aquel elemento autónomo y claramente identificable propio de la arquitectura moderna, parece entonces haber desaparecido. El objetivo del presente artículo es señalar de que modón cla cluso del vidrio como material estructural en la y el sistema estructural que ha caracterizado a la arquitectura moderna.

PALABRAS CLAVE: cerramiento; estructura; vidrio; transparencia; tectónica.

Persona de contacto: albert.albareda@upc.edu. Departamento de Tecnología de la Arquitectura de la Escuela Técnica Superior de Arquitectura de Barcelona (UPC).

esde la antigüedad hasta el siglo XIX la estructura portante tiene un papel fundamental en la definición del espacio arquitectónico. Sin embargo, la irrupción del acero como material estructural y del vidrio como cerramiento transparente durante el siglo XIX permite disociar ambas funciones en dos sistemas constructivos totalmente diferentes. La arquitectura moderna, a través de la cubierta plana y la planta libre, hace de esta dicotomía su estandarte: nunca antes en la historia de la arquitectura, la estructura vertical había adquirido una autonomía total con respecto al cerramiento, pudiendo éste ser totalmente transparente.

La voluntad de disponer de cerramientos de vidrio de cada vez mayores dimensiones, hace que la industria del vidrio evolucione considerablemente a finales del siglo XX. Esta evolución tecnológica deriva en una mejora importantísima de las prestaciones mecánicas del vidrio, que permite empezar a plantear su uso como elemento estructural: por primera vez en la historia de la construcción es posible emplear un material transparente como estructura. Estructura y cerramiento vuelven a unificarse como en la antigüedad, pero esta vez lo hacen en un elemento totalmente transparente. La estructura, entendida como aquel elemento autónomo y claramente identificable propio de la arquitectura moderna, parece entonces haber desaparecido.

El objetivo del presente artículo es señalar de qué modo afecta el uso del vidrio como material estructural en la relación de dicotomía entre el sistema de cerramiento espacial y el sistema estructural que ha caracterizado a la arquitectura moderna.

La estructura como elemento portante de definición espacial

La arquitectura es el arte de la delimitación espacial, en respuesta a unas necesidades programáticas. Desde un punto de vista tectónico, esta delimitación espacial puede producirse horizontalmente, definiendo un recinto por medio de un cerramiento vertical, o verticalmente, cubriendo el espacio mediante un techo. En ambos casos, dicha delimitación espacial se consigue por oposición entre la materia y su ausencia: a la materia que, dispuesta de acuerdo a las leyes de la gravedad, construye el espacio, la denominamos estructura; y a la ausencia de materia resultante de dicha operación arquitectónica la llamamos espacio. La arquitectura, concebida como realidad constructiva, queda entonces indisolublemente vinculada la estructura o "actividad de construir" (del latín Structus -construir- y -ura actividad-)

Desde un punto de vista estrictamente estático, la dificultad estructural que supone la delimitación del espacio puede tener un carácter muy distinto. A lo largo de la historia de la arquitectura, los elementos estructurales verticales -muros, columnas, etc.- han acostumbrado a ser retos estructurales menores que las cubriciones espaciales. Esto se debe, fundamentalmente, a la coincidencia o no de la dirección del elemento con la dirección en la que se manifiesta cotidianamente la gravedad. Los soportes tienen una naturaleza eminentemente vertical que los alinea con la dirección natural -vertical hacia abajo- de las cargas gravitatorias. En cambio, en los elementos horizontales se produce un desajuste provocado po la necesidad de cubrir el espacio y la obligatoriedad de encauzar horizontalmente las cargas verticales que aparecen por el propio peso del elemento. Este desajuste ha supuesto tradicionalmente un reto estructural muy significativo.

Este desafío ha obligado siempre a comprender profundamente el comportamiento mecánico de los materiales disponibles para la construcción. El distinto origen natural de los materiales térreos como la piedra o el adobe- y la madera, explica su como la piedra o el adobe- y la madera, explica su diversa idoneidad de aplicación estructural. Desde los inicios, la corteza terrestre se ha visto sometida a esfuerzos de compresión titánicos por efecto de la acción de la gravedad. De ahí la elevada resistencia de la piedra sometida a esfuerzos de compresión, siendo un material idóneo para la construcción de elementos estructurales verticales. Sin embargo, sus capacidades a compresión no son correspondidas a tracción, y por tanto, altamente vulnerable también a esfuerzos de flexión. Es por ello que su aplicación en elementos de cubrición a flexión presenta importantes limitaciones. En cambio, las ramas y los troncos de los árboles deben poder resistir, además de su propio peso, las deformaciones por flexión provocadas por acciones perpendiculares a su eje, como por ejemplo las ejercidas por el viento. Ello explica la elevada resistencia de la madera sometida a esta clase de esfuerzos, convirtiéndose así en un material idóneo para la construcción de elementos estructurales horizontales sometidos a esfuerzos de flexión por su peso propio. Además, se da la circunstancia de que la densidad de la piedra, en general, es siempre mucho mayor que la de la madera: al ser ésta un material mucho más ligero que la primera, resulta mucho más adecuada en la construcción de cualquier cubrición espacial desde un punto de vista puramente estructural.

Estas diferentes cualidades de los materiales estructurales impusieron durante milenios una lógica constructiva que Aristóteles resumió con elocuencia: $E$ I muro ha sido hecho por necesidad, porque lo pesado se desplaza naturalmente hacia abajo y lo ligero hacia arriba, esto es, porque las piedras y los cimientos se ponen abajo, encima los ladrillos por ser más ligeros, y en lo más alto las maderas por ser todavía más ligeras ${ }^{1}$. Esta limitación afectó a obras tan distintas en su concepción y tan distantes en el tiempo como el Stonehenge o el Partenón, arquitecturas en las que los soportes poseen una robustez excesiva en relación al espacio libre que son capaces de salvar los dinteles pétreos.

Tal como apunta Choisy, para ir más allá, para aumentar todavía más la resistencia de los arquitrabes o para espaciar más los apoyos, había que cambiar radicalmente el sistema de construcción, era preciso renunciar a los dinteles monolíticos y recurrir, bien a las bóvedas de hormigón, bien a las de cantería². En efecto, el uso de las formas estructurales curvas de cubrición espacial, como fue primero la falsa cúpula y, después, el arco de descarga, con sus variantes espaciales de traslación (bóveda) o rotación (cúpula), constituyó una de las más importantes aportaciones de la construcción romana a la historia de la arquitectura, ya que permitió resolver la cubrición espacial empleando materiales sometidos principalmente a esfuerzos de compresión.

Desde la irrupción del sistema abovedado romano y durante aproximadamente dos mil años, la fusión entre el sistema estructural y el sistema de delimitación espacial se mantuvo como una constante de la arquitectura monumental. Así ocurre en construcciones aparentemente tan diferentes en su concepción técnica y estilística como el Pantheon de Roma o la Catedral de Chartres: tanto los masivos y gruesos muros ciegos del primero, que se van curvando hacia el centro de la planta para convertirse gradualmente en delgadas paredes de una cúpula, como las esbeltas y ahuecadas estructuras pétreas del segundo, comparten un mismo objetivo, que no es otro que el de encauzar la gravedad para confinar un espacio interior. Arquitectura y estructura se convierten entonces en conceptos indisociables: lo que porta, cierra y cubre es lo mismo.

Hacia la independencia estructural: aceptación de la estructura como sistema autónomo

Todo cambia durante el siglo XIX con la producción industrial de hierro y acero de aplicación estructural. Además de poseer enormes capacidades mecánicas, estos metales funcionan simétricamente a tracción y compresión, hecho que los convierte en materiales de construcción excepcionales. Estas nuevas posibilidades estructurales, en combinación con la aparición de nuevos métodos de cálculo, hicieron posible la construcción de estructuras muy esbeltas -en celosía- de luces hasta entonces inimaginables, que se combinaron con grandes cerramientos de vidrio. De este modo, no sólo se consiguió inundar de luz el espacio interior, sino que se consiguió desvincular definitivamente el sistema estructural -metálico- de

\section{v FIG. 1 (IZQ). Charles Louis Ferdinand Dutert y Victor Contamin: Palais des Machines (1889, París). Vacío interior de la
gran nave central del Palais des Machines. GIEDION, Sigfried; GEORGIADIS, Sokratis: Building in France, building in iron,
building in ferro-concrete. Santa Monica: Getty Center for the History of Art and the Humanities, 1995, p. 140.
FIG. 2 (DCHA). Le Corbusier. Estructura Dom-lnó (1914-1915).}
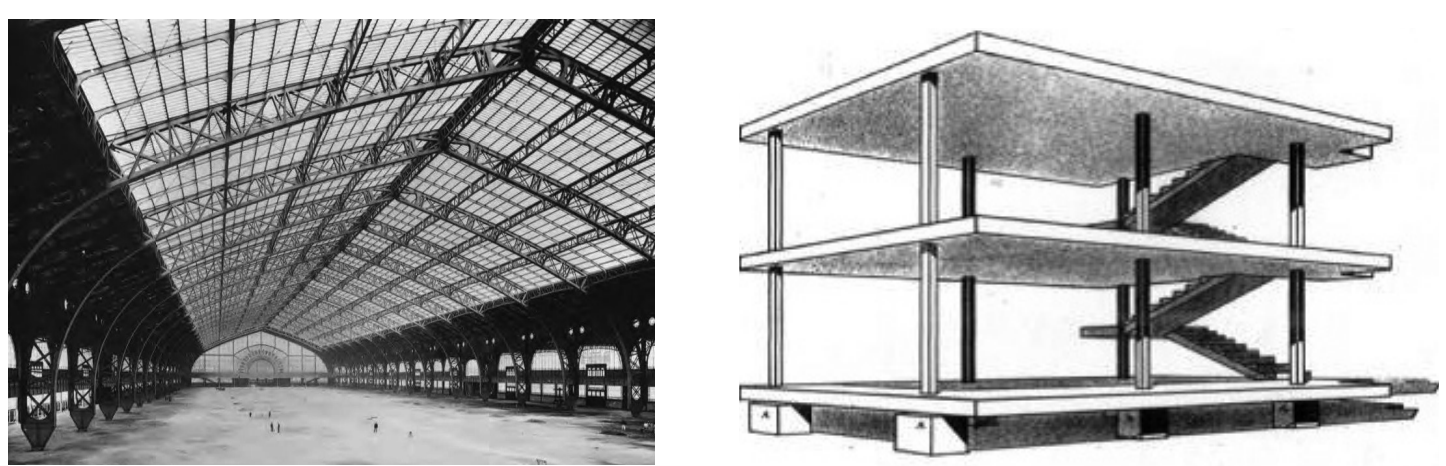


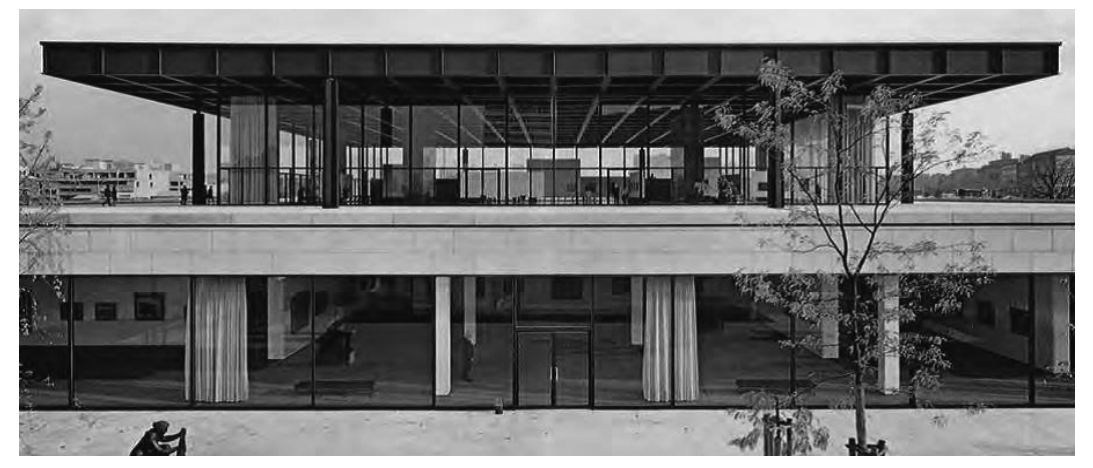

FIG. 3 (IZQ). Mies van der Rohe: Neue Nationalgalerie (1962-1968, Berlín FG. 3 (IZQ). Mies van der Rohe: Neue Nationalgalerie (1962-1968, Berlin).
Visión desde el patio. www.vizcayado.blogspot.com
FIG. 4 (DCHA). Giuseppe Terragni: Dateum, Sala del Paraíso (1941, Roma). Visión desde el patio. ARNUNCIO PASTOR, Juan Carlos: Peso y levedad: notas sobre la
gravedad a partir del Danteum. Barcelona: Fundación Caja de Arquitectos, 2007

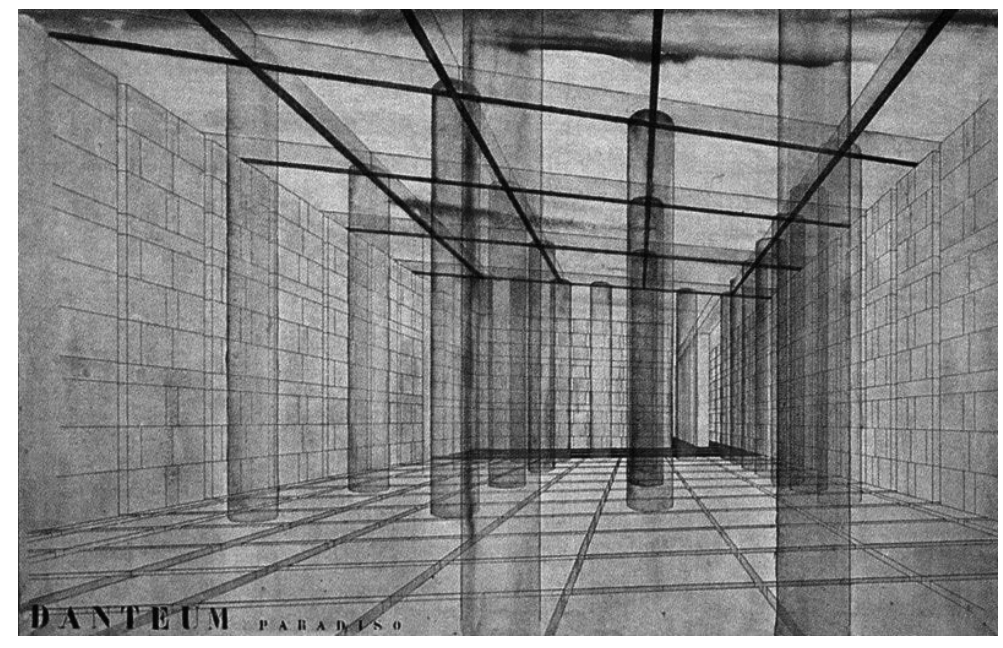

un sistema de cerramiento espacial -vítreo- cuya transparencia no hizo más que enfatizar la autonomía de ambos elementos. Obras tan diferentes como la Palm House de los Kew Gardens de Londres, el Crystal Palace de Paxton o el Palais des Machines de la Exposición Universal de París de 1889 (FIG. 1) expresan en mayor o menor medida esta circunstancia.

Durante el siglo XX, asimiladas ya las capacidades estructurales del acero, se descubre la posibilidad de abandonar las rígidas leyes de la estática gráfica -principalmente abovedada- en favor de los sistemas de cubrición planos, mucho más acordes a las necesidades de la construcción edilicia. En 1915 Le Corbusier expone con elocuencia este ideal en su Estructura Dom-Inó (FIG. 2): el techo plano se convierte en un requisito fundamental de la planta libre, es decir, de la independencia entre la estructura vertical (puntual) y unos elementos de delimitación vertical interiores (particiones) y exteriores (fachada) que, idealmente, deben poder disponerse libremente.

La estructura se afianza como un sistema autónomo: ya no se trata de muros aligerados que sustentan bóvedas apuntadas y que, al mismo tiempo, delimitan el espacio; se trata de esbeltos soportes que soportan retículas planas, bajo las cuales puede fluir libremente el espacio. A fin de llegar a una ecuación de las exigencias de espacio y estructura, Le Corbusier y Mies van der Rohe se habían visto llevados a postular la independencia funcional, es decir, la independencia de los tabiques de las columnas, de modo que, (...) el Estilo Internacional puede ser interpretado como derivado del supuesto de una existencia independiente ambos, que seguirían leyes distintas. (...) En el Estilo Internacional una estructura autónoma perfora un espacio libremente abstraído, actuando no como su forma definitoria, sino como su puntuación. En el Estilo Internacional, por tanto, no hay fusión de espacio y estructura y, al final, cada uno continúa siendo un componente identificable, mientras la arquitectura no es concebida como confluencia de ambos sino más bien como su oposición dialéctica, como una especie de debate entre ellos ${ }^{3}$. Colin Rowe expone con claridad la dicotomía existente entre el espacio moderno y la estructura que lo hace posible. La estructura vertical moderna sí participa en la definición del espacio pero, en vez de confinarlo, es concebida para hacer realidad una nueva espacialidad más libre, abierta y fluida. Afirmar que la estructura moderna no participa de la definición del espacio arquitectónico supondría no entender que el idea espacial que persiguió la arquitectura moderna, un espacio interior en continuidad con el exterior, fue muy diferente al espacio interior confinado que caracterizó a la arquitectura durante los dos mil años anteriores: lógicamente, la relación de la estructura con el espacio debía ser muy diferente a la que hasta entonces se había producido.

Lógicamente, la posibilidad de fabricar industrialmente vidrios planos de gran formato permite construir cerramientos de fachada transparentes. La intrínseca transparencia de estos cerramientos vítreos permite separar homeostáticamente el espacio interior del exterior sin impedir una casi total transparencia exterior ambos espacios, sólo comprometida por los eventuales brillos y reflejos de la propia superficie acristalada. El cerramiento moderno altera, entonces, la condición de frontera entre el interior y el exterior que había tenido hasta entonces el muro pues, aunque mantiene la separación física entre el espacio interior y el exterior, al mismo tiempo construye una relación visual directa entre ambos espacios.
Esta transparencia no sólo permite obtener una gran conexión visual y espacial entre interior y exterior: permite, también, explicitar la autonomía que se produce entre el cerramiento vertical (fachada) y la estructura vertical (soporte). Nunca antes la estructura como tal se había identificado con tanta claridad como sistema independiente del hecho espacial. La expresión de esta clara autonomía estructural se convierte casi en un fin en sí mismo, una característica definitoria y definitiva de la arquitectura moderna. Los pilotis de la Villa Savoye Le Corbusier o los pilares cruciformes de la Neue National Galerie de Mies va der Rohe (FIG. 3) no son casuales; permiten evidenciar la claridad del sistema estructural más allá de lo estrictamente necesario.

Por primera vez en la historia de la construcción se consigue, también, invertir el principio constructivo aristotélico según el cual la cubierta debe ser necesariamente más ligera que el soporte. Mientras que históricamente el muro se concibió como un pesado elemento portante, y se buscó siempre la máxima ligereza en la cubierta, en la construcción moderna el elemento portado -la cubierta- adquiere una presencia y una masa mucho mayor que la del sistema portante -los soportes-, que ha reducido a su mínima expresión gracias a la enorme capacidad mecánica del acero. La disposición de una frágil fachada acristalada, independiente del sistema estructural, no hace más que enfatizar esta ambigua condición gravitatoria de la cubierta, realmente pesada pero aparentemente liviana.

\section{La reunificación del sistema estructural} y el cerramiento espacial a través de la transparencia

La cualidad del espacio depende, en gran medida, de las propiedades de la luz que lo configura. El carácter arquitectónico del espacio viene definido por la adecuada relación que se establece entre la luz y la sombra, definida por la disposición y la naturaleza de la materia que compone los límites del propio espacio. Se descubre entonces que, en tanto que opaca, la materia a la que nos referimos como estructura posee la capacidad de ordenar, con su sola presencia, la luz que configura el espacio. A lo largo de la historia, la arquitectura ha indagado en diferentes modos de disponer la materia pesada y opaca con la que define sus límites para definir, así, un espacio adecuadamente iluminado.
Durante el siglo XX la voluntad de disponer de fachadas de vidrio con paños sin carpintería cada vez de mayores dimensiones obliga la industria a buscar soluciones tecnológicas (aumento de los formatos disponibles, mejora de las posibilidades de laminación, mejora de los procesos de templado, innovación en el uso de butirales estructurales, etc.) que permitan al vidrio soportar los esfuerzos y las , principalmente, por las acciones gravitatorias y el viento. En este sentido, se explora el uso de costillas de vidrio, la combinación con estructuras de acero tensadas y las uniones metálicas o mediante siliconas estructurales.

A finales de siglo se descubre que todo este desarrollo tecnológico, orientado inicialmente a aumentar las capacidades mecánicas de los cerramientos transparentes, hace posible empezar a pensar en la posibilidad de utilizar el vidrio como material estructural. Por primera vez se abre la posibilidad de construir estructuras literalmente transparentes, una realidad que hasta entonces había sido siempre una utopía. No obstante, los primeros elementos estructurales enteramente de vidrio que se plantean son soportes, estructuras básicamente comprimidas, puesto que el vidrio es un material de origen pétreo que una buena resistencia a compresión pero que resiste relativamente mal a tracción. Además, su fragilidad hace que sea un material extremadamente delicado a efectos estructurales, puesto que la falta de ductilidad impone unos coeficientes de seguridad altos.

La industria del vidrio permite, a partir de la década de los ochenta del siglo pasado, empezar a explorar las capacidades estructurales del vidrio en pequeñas construcciones. Aunque el objetivo es conseguir estructuras totalmente acristaladas, como las que imaginó en 1941 Giuseppe Terragni para la Sala del Paraíso del Danteum (FIG. 4), estas primeras tentativas dan lugar a sistemas mixtos de vidrio y acero. El uso del vidrio se reserva para los elementos sometidos principalmente a compresión -como los soportes-, donde se confía más en las características mecánicas del material. En cambio, los elementos estructurales sometidos a esfuerzos de tracción o flexión importantes, se resuelven con acero. El Sonsbeek Pavilion en Arnhem (FIG. 5), proyectado en 1986 por Benthem Crouwel Architects, es un buen ejemplo.

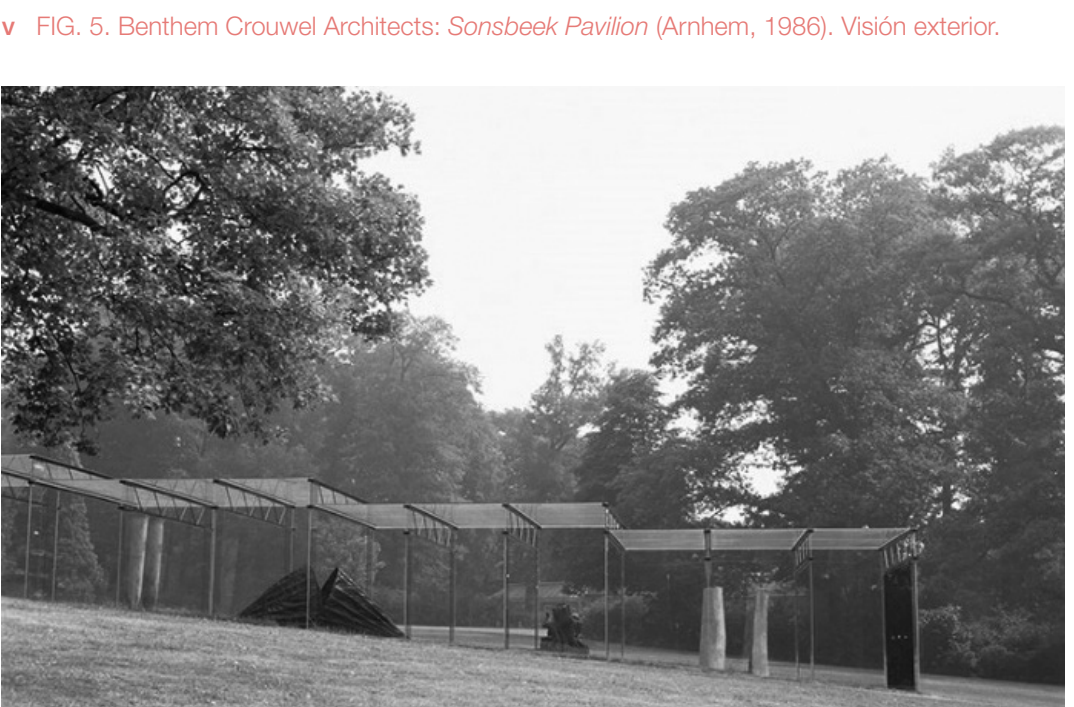


Pero el ideal que persiguen estas construcciones sigue siendo el que en su momento dibujó Terragni: vigas y columnas totalmente de vidrio. El uso de vidrios templados laminados, siliconas estructurales y uniones metálicas puntuales de tamaño cada vez más reducido, permite erigir estructuras totalmente acristaladas en las que el acero desempeña un papel fundamental pero con un impacto visual mínimo. La ampliación del Broadfield House Glass Museum en Dudley, proyectado en 1994 por Design Antenna, con sus fijaciones de acero ocultas en la base de los soportes y los apoyos en el muro, explora con éxito esta vía de trabajo. Pero, sin duda, la Apple Store de la Quinta Avenida de Nueva York, diseñada por el arquitecto Bohlin Cywinsk Jackson y la ingeniería Eckersley O'Callahan, evidencia la rápida evolución que experimenta la industria del vidrio: en 2006 se erige un primer cubo de vidrio de 10 metros de lado, que se cubre con un emparrillado de $5 \times 5$ vigas apoyadas sobre 20 soportes de vidrio laminado mayor dimensión del proyecto, utilizados laminarmente como elementos de rigidización del entramado estructural, miden 2,0 × 3,3 metros. En 2011, a petición de Apple, el mismo equipo de diseño sustituye aquella estructura por otra que conserva las dimensiones, pero que se resuelve con un emparrillado de sólo $2 \times 2$ vigas y que utiliza ya vidrios de $3,2 \times 10,0$ metros como cerramiento estructural (FIG. 7). En ambos casos se utilizan pequeñas uniones metálicas para hacer solidarios los diferentes elementos vítreos.

Esta vía de trabajo llevaba implícita la idea de que la construcción de estructuras de vidrio debe perseguir una transparencia total, tanto en cubierta como en fachada. Pero esta idea lleva implícita una serie de limitaciones. Desde un punto de vista técnico, la voluntad de construir cubiertas totalmente acristaladas obliga a resolver el elemento de cubrición con vidrio, un material de densidad muy similar a la piedra (unos $2.500 \mathrm{~kg} /$ $\mathrm{m} 3$ ); teniendo en cuenta que el vidrio no trabaja bien a flexión, se impone el uso de jácenas de canto de vidrio que, además de dar lugar a complicadísimas uniones metálicas, concentran enormes solicitaciones en determinados puntos de la estructura vertical, sometiendo al vidrio en esas zonas a grandes tensiones. Se descubre entonces que debe recurrirse a una tipología estructural de vigas y pilares que, además de tener una limitación de luz importante, parece estar en disonancia con las características mecánicas de propio material. Además, todo este esfuerzo va dirigido a lograr una transparencia horizontal en la cubierta que, además de no aportar ningún valor espacial (ya que se consigue un objeto arquitectónico cuyo interior, a efectos de luz, parece un exterior), acarrea graves problemas energéticos; algo que, por cierto, ya se había descubierto a lo largo del siglo XIX a propósito de las construcciones de acero y vidrio.

Todo ello lleva a pensar que para encontrar el modo correcto de hacer funcionar al vidrio es necesario comprender su naturaleza laminar. A partir de ese momento se orienta la investigación proyectual hacia el diseño de objetos arquitectónicos formados por estructuras verticales de vidrio que soportan cubiertas planas opacas. Este planeamiento permite mantener el ideal de la fluidez horizontal espacial y visual propio de la arquitectura moderna, contrapuntada por la presencia de una cubierta opaca que controla el exceso de luz; y todo ello eliminando, al mismo tiempo, la presencia de cualquier elemento estructural vertical.

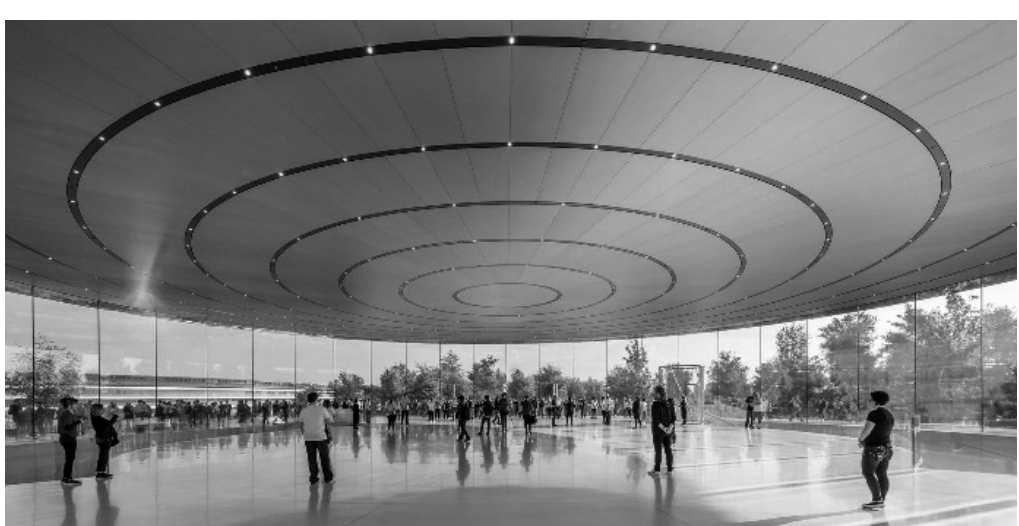

FIG. 8. Foster and Partners + Eckersley O'Callahan: Steve Jobs Theater.

Las primeras pruebas que se hacen para combinar estructuras verticales de vidrio con cubiertas opacas surgen con el cambio de siglo. La estructura vertical, de vidrio, se concibe por lo general como un conjunto de vidrios empotrados en su base con el objetivo aumentar su inercia en el sentido más desfavorable de la lámina, dispuestos en planta en sentidos perpendiculares para arriostrarse entre $\mathrm{si}$. Esta estructura soporta un emparrillado horizontal que se intenta hacer lo más ligero posible para no cargar en exceso los soportes, sometidos ya de por sí a grandes esfuerzos debido a su enorme delgadez. Un buen ejemplo de este planteamiento es la Escuela Técnica de Rheinbach, proyectado en el año 2000 por los arquitectos Jürgen Marquardt y Jörg Hieber y la ingeniería Ludwig \& Weiler.

El material estructural más utilizado en la cubierta es el acero, pues se le vincula más a la construcción ligera que el hormigón. En efecto, su uso en la cubierta permite soslayar las dificultades técnicas y mecánicas que presentan las uniones entre vidrios, pero su elevada densidad $(7.850 \mathrm{~kg} / \mathrm{m} 3)$ penaliza en exceso los soportes de vidrio, especialmente cuando se intentaban alcanzar luces importantes. Aunque se intente reducir al mínimo sus secciones, el peso propio que resulta al limitar las deformaciones de estas estructuras de cubrición deviene siempre excesivamente alto. Este problema se soluciona al descubrir la posibilidad de construir cubiertas de fibra de carbono, un material ampliamente utilizado en la industria automovilística, naval y aeronáutica por tener propiedades mecánicas similares al acero, una baja densidad $(1.750 \mathrm{Kg} / \mathrm{m} 3)$, una elevada resistencia a los agentes externos y cambios de temperatura, y buenas prestaciones como aislamiento térmico. El uso de este material en cubierta supone, como ocurría en la era preindustrial, que la cubierta vuelve a pesar menos que el soporte, una situación que desde el siglo XIX podía obviarse sin demasiado esfuerzo.

Foster and Partners, en colaboración con la ingeniería Eckersley O'Callahan, emplea esta tecnología en su primer proyecto para Apple, el Apple Zorlu Center erigido en 2014 en Estambul. Una gran linterna de vidrio de 10x10 metros en planta se convierte, igual que en la Apple Store de la quinta avenida, en la parte visible de un espacio de venta al público ubicado en planta sótano. En un ejercicio proyectual de gran elegancia en el que la técnica se refina tanto que parece desaparecer, esta gran linterna se resuelve únicamente mediante cuatro vidrios de 10,0 × 3,2 metros y una cubierta de fibra de carbono de $10 \times 10$ metros con un espesor de sólo $175 \mathrm{~mm}$ en el centro y $50 \mathrm{~mm}$ en los bordes.

Sólo tres años más tarde, el mismo equipo es capaz de diseñar y construir el Steve Jobs Theater en California (FIG. 8), una estructura circular de vidrio estructural de 7 metros de alto y 41 metros de diámetro (una dimensión inferior en sólo dos metros y medio a la del diámetro interior del Pantheon de Roma) que soporta una gran cubierta de fibra de carbono de 80 toneladas. A nivel técnico, el uso estructural del vidrio supone una proeza estructural hasta el momento impensable.

Durante los últimos cinco años el equipo formado por Foster and Partners y Eckersley O'Callahan ha indagado en las posibilidades estructurales del vidrio hasta límites insospechados pocos años atrás. A través de su obra construida, han demostrado que, empleado como una lámina vertical, el vidrio deviene un material estructural más.

Se constata entonces lo siguiente: el uso estructural de láminas de vidrio que conforman, a su vez, la fachada del edificio, supone la reunificación del cerramiento que delimita el espacio y la estructura resistente en un mismo elemento. En este sentido, se vuelve a un modelo previo al que preconizó la modernidad y que caracterizó a la arquitectura desde la antigüedad hasta el siglo XIX, en el que el muro aunaba la función del cerramiento y la estructura. Ello podría considerarse, de alguna manera, una cierta involución, en tanto que se retorna a la unidad de cerramiento y estructura previa a la modernidad; pero cierto es, también, que en este nuevo escenario se mantiene aquella transparencia que defendió la modernidad. Este elemento que aúna estructura y cerramiento no posee ni el espesor ni la opacidad que se le presupone implícito al muro. Se trata, más bien, de un paramento de gran esbeltez y máxima transparencia, que por nuestra educación técnica y visual identificamos casi automáticamente con el cerramiento y no con la estructura. Esto hace que la estructura, entendida como aquel sistema autónomo y claramente identificable que caracterizó a la arquitectura moderna, parezca haber desaparecido en este tipo de arquitecturas. Pero lo que realmente ha ocurrido es que se ha tornado transparente y ha sido absorbida de nuevo por un elemento, el cerramiento, función portante. Y esto no es una cuestión menor. 1 "Física”, Libro Il, 200a. ARISTÓTELES; ECHANDÍA, Guillermo
R. (ed): Física/Aristóteles. Madrid: Gredos, 1995, pp. 168-169. 2 CHOISY, Auguste; HUERTA FERNÁNDEZ, Santiago (ed); GIRÓN SIERRA, Francisco Javier (ed): El Arte de construir en Roma. Madrid: CEHOPU, 1999, p. 109. ${ }^{3}$ ROWE, Colin: Manierismo y arquitectura moderna y otros ensayos. Barcelona:
de Chicago, 1956.

OSCAR LINARES DE LA TORRE es Doctor Arquitecto y Profesor Asociado del Departamento de Proyectos Arquitectónicos de la ETSAB, Universidad Politécnica de Catalunya (UPC).

ALBERT ALBAREDA VALLS es Doctor Arquitecto y Profesor Asociado del Departamento de Tecnología de la Arquitectura de la ETSAB, Universidad Politécnica de Catalunya (UPC). 